\title{
Low-frequency electrostatic wave in a metallic electron-hole-ion plasma with nanoparticles
}

\author{
P. K. SHUK L A ${ }^{1}$ and G. E. MOR F I L L ${ }^{2}$ \\ ${ }^{1}$ Institut für Theoretische Physik IV, Ruhr-Universität Bochum, D-44780 Bochum, \\ Germany \\ Also at Department of Physics, Umeå University, SE-90187 Umeå, Sweden; Scottish \\ Universities Physics Alliance (SUPA), Department of Physics, University of Strathclyde, \\ Glasgow G4 0NG, Scotland, United Kingdom; Science and Technology Facilities Council \\ (STFC) Centre for Fundamental Physics, Rutherford Appleton Laboratory, Harwell \\ Science and Innovation Centre, Chilton, Didcot, Oxon, OX11 OQX, United Kingdom; \\ GoLP/Instituto de Plasmas e Fusão Nuclear, Instituto Superior Técnico, 1049-001 Lisboa, \\ Portugal; School of Physics, University of KwaZulu-Natal, 4000 Durban, South Africa \\ (ps@tp4.rub.de) \\ ${ }^{2}$ Max-Planck Institut für extraterrestrische Physik, D-85740 Garching, Germany
}

(Received 3 April 2009 and accepted 20 April 2009, first published online 12 June 2009)

\begin{abstract}
We investigate the dispersion property of a low-frequency electrostatic wave in a dense metallic electron-hole-ion plasma with nanoparticles. The latter are charged due to the field emission, and hence the metallic nanoparticles/nanotubes can be regarded as charged dust rods surrounded by degenerate electrons and holes, and non-degenerate ions. By using a quantum hydrodynamic model for the electrons and holes, we obtain the electron and hole number density perturbations, while the ion and dust rod number density perturbations follow the classical expressions. A dispersion relation for the low-frequency electrostatic wave in our multispecies dense metallic plasma is derived and analyzed. The possibility of exciting non-thermal electrostatic waves is also discussed.
\end{abstract}

Carbon nanotubes [1] were discovered around 18 years ago. Since then there have been a great deal of experimental $[2,3]$ and theoretical $[4,5,7-10]$ investigations of localized collective electronic excitations (plasmon modes) in single- and multiwalled carbon nanotubes (CNTs). The knowledge of dispersion properties of numerous plasmonic modes is essential for determining the parameters that control electronic structures of CNTs.

The CNTs can be metallic or semiconducting depending on their radius and the geometric angles. When CNTs are held in plasmas, one encounters charging of CNTs due to the electric field emission $[11,12]$. Henceforth, CNTs in a dense metallic plasma can be regarded as an ensemble of charged nanoparticles surrounded by degenerate electrons and holes, as well as non-degenerate ions. Collective interactions between the plasma and charged nanoparticles can give rise to new wave modes in a multi-species dense metallic plasma.

In this letter, we report on the existence of a low-frequency electrostatic wave and its instability in a metallic plasma. The latter is composed of degenerate electrons 
and holes, as well as ions, charged nanoparticles, and immobile neutral atoms. At equilibrium, the quasi-neutrality condition is [13-16] $q_{\mathrm{i}} n_{\mathrm{i} 0}+e n_{\mathrm{h} 0}=e n_{\mathrm{e} 0}+q_{\mathrm{d}} n_{\mathrm{d} 0}$, where $q_{\mathrm{i}}$ is the ion charge, $n_{\mathrm{j} 0}$ is the unperturbed number density of the particle species $j$ (j equals i for ions, e for electrons, $\mathrm{h}$ for holes, and $\mathrm{d}$ for charged dust CNTs), $q_{\mathrm{d}}=Z_{\mathrm{d}} e\left(-Z_{\mathrm{d}} e\right)$ for negative (positive) nanoparticles, $Z_{\mathrm{d}}$ is the number of charges on nanoparticles, and $e$ is the magnitude of the electron charge. In the presence of the low-frequency space charge electric field $-\nabla \phi$, where $\phi$ is the self-consistent electrostatic potential, the electron and hole density perturbations, $n_{\mathrm{e} 1}\left(\ll n_{\mathrm{e} 0}\right)$ and $n_{\mathrm{h} 1}\left(\ll n_{\mathrm{h} 0}\right)$, are obtained from the inertialess electron and hole momentum equations, respectively,

$$
0=e n_{\mathrm{e} 0} \nabla \phi-\nabla p_{\mathrm{e} 1}+\frac{\hbar^{2}}{4 m_{\mathrm{e}}} \nabla \nabla^{2} n_{\mathrm{e} 1}
$$

and

$$
0=-q_{\mathrm{i}} n_{\mathrm{h} 0} \nabla \phi-\nabla p_{\mathrm{h} 1}+\frac{\hbar^{2}}{4 m_{\mathrm{h}}} \nabla \nabla^{2} n_{\mathrm{h} 1},
$$

where $m_{\mathrm{e}}\left(m_{\mathrm{h}}\right)$ is the electron (hole) mass, and $\hbar$ is the Planck constant divided by $2 \pi$. The second and third terms on the right-hand side of (1) and (2) are associated with the quantum statistical pressure laws $[17-19]$ (e.g. $p_{\mathrm{el}, \mathrm{hl}}=\left(3 n_{\mathrm{e} 0, \mathrm{~h} 0} / 8 \pi\right)^{2 / 3}$ $\left(4 \pi^{2} \hbar^{2} / 3 m_{\mathrm{e}, \mathrm{h}}\right) n_{\mathrm{el}, \mathrm{hl}}$ for non-relativistic degenerate electrons and holes) and the quantum Bohm force [17-26] associated with quantum electron/hole tunneling effects due to the finite width of the electron and hole wave functions, respectively.

The electrons and holes are coupled with the ions and charged nanoparticles via the space charge electric field. The ion number density perturbation $n_{\mathrm{il}}\left(\ll n_{\mathrm{i} 0}\right)$ is obtained from the ion continuity and ion momentum equations

$$
\frac{\partial n_{\mathrm{il}}}{\partial t}+n_{\mathrm{i} 0} \nabla \cdot \mathbf{u}_{\mathrm{i}}=0
$$

and

$$
\frac{\partial \mathbf{u}_{\mathrm{i}}}{\partial t}-\frac{q_{\mathrm{i}}}{m_{\mathrm{i}}} \nabla \phi=0
$$

where $\mathbf{u}_{\mathrm{i}}$ and $m_{\mathrm{i}}$ are the ion velocity and the ion mass, respectively.

The nanoparticle number density perturbation $n_{\mathrm{dl}}\left(\ll n_{\mathrm{d} 0}\right)$ is determined from the continuity and momentum equations [27]

$$
\frac{\partial n_{\mathrm{d} 1}}{\partial t}+n_{\mathrm{d} 0} \nabla \cdot \mathbf{u}_{\mathrm{d}}=0
$$

and

$$
\frac{\partial \mathbf{u}_{\mathrm{d}}}{\partial t}+\frac{q_{\mathrm{d}}}{m_{\mathrm{d}}} \nabla \phi=0
$$

where $\mathbf{u}_{\mathrm{d}}$ and $m_{\mathrm{d}}$ are the nanoparticle velocity and the nanoparticle mass, respectively.

The self-consistent potential is given by

$$
\phi\left(\mathbf{r}_{s}, t\right)=\int d s^{\prime} \frac{\left[q_{\mathrm{i}} n_{\mathrm{il}}\left(\mathbf{r}_{s}^{\prime}, t\right)+e n_{\mathrm{h} 1}\left(\mathbf{r}_{s}^{\prime}, t\right)-e n_{\mathrm{e} 1}\left(\mathbf{r}_{s}^{\prime}, t\right)+q_{\mathrm{d}} n_{\mathrm{d} 1}\left(\mathbf{r}_{s}^{\prime}, t\right)\right]}{\left|\mathbf{r}_{s}-\mathbf{r}_{s}^{\prime}\right|},
$$


where $d s^{\prime} R d \varphi d z^{\prime}$ and $R$ is the radius. By using the expansion of the Coulomb potential, $1 /\left|\mathbf{r}_{s}-\mathbf{r}_{s}^{\prime}\right|$, in cylindrical coordinates, one can write $[10,28]$

$$
\phi\left(\mathbf{r}_{s}, t\right)=\sum_{m=-\infty}^{\infty} \int_{\infty}^{\infty} \frac{d k}{(2 \pi)^{2}} G(R, k, m) \int d s^{\prime} \rho \exp \left[i k\left(z-z^{\prime}\right)+i m\left(\varphi-\varphi^{\prime}\right)\right],
$$

where $k$ is the longitudinal (axial) wave number and $m$ is the discrete azimuthal quantum number. We have denoted $\rho=q_{\mathrm{i}} n_{\mathrm{il}}\left(\mathbf{r}_{s}^{\prime}, t\right)+e n_{\mathrm{h} 1}\left(\mathbf{r}_{s}^{\prime}, t\right)-e n_{\mathrm{e} 1}\left(\mathbf{r}_{s}^{\prime}, t\right)+$ $q_{\mathrm{d}} n_{\mathrm{d} 1}\left(\mathbf{r}_{s}^{\prime}, t\right)$ and $G=4 \pi I_{m}(k R) K_{m}(k R)$, where $I_{m}$ and $K_{m}$ are the cylindrical Bessel functions of order $m$.

We shall apply the Fourier transformation [10]

$$
F\left(\mathbf{r}_{s}, t\right)=\sum_{m=-\infty}^{\infty} \int_{\infty}^{\infty} \frac{d k}{(2 \pi)^{2}} F(R, k, m, \omega) \int d s^{\prime} \exp \left[-i \omega t+i k\left(z-z^{\prime}\right)+i m\left(\varphi-\varphi^{\prime}\right)\right]
$$

where $F$ is any physical quantity and $\omega$ is the frequency, on the governing (1)-(6). From (1) and (2), we then have

$$
\begin{gathered}
n_{\mathrm{e} 1}=\frac{4 \pi n_{\mathrm{e} 0} e m_{\mathrm{e}} \phi}{\hbar^{2}\left(k_{m}^{2}+k_{F \mathrm{e}}^{2}\right)}, \\
n_{\mathrm{h} 1}=-\frac{4 \pi n_{\mathrm{i} 0} e m_{\mathrm{h}} \phi}{\hbar^{2}\left(k_{m}^{2}+k_{F \mathrm{~h}}^{2}\right)},
\end{gathered}
$$

where $k_{F \mathrm{e}, F \mathrm{~h}}^{2}=16 \pi^{2}(3 / 8 \pi)^{2 / 3} n_{\mathrm{e} 0, \mathrm{~h} 0}^{2 / 3}, k_{m}^{2}=k^{2}+m^{2} / R^{2}$.

The ion and nanoparticle number density perturbations, deduced from (4)-(6) by using (9), are, respectively,

$$
\begin{aligned}
n_{\mathrm{il}} & =\frac{q_{\mathrm{i}} n_{\mathrm{i} 0} k_{m}^{2} \phi}{m_{\mathrm{d}} \omega^{2}}, \\
n_{\mathrm{d} 1} & =\frac{n_{\mathrm{d} 0} q_{\mathrm{d}} k_{m}^{2} \phi}{m_{\mathrm{d}} \omega^{2}} .
\end{aligned}
$$

The Fourier transformed potential reads [10]

$$
\phi=G R^{2}\left(q_{\mathrm{i}} n_{\mathrm{i} 1}+e n_{\mathrm{h} 1}-e n_{\mathrm{e} 1}+q_{\mathrm{d}} n_{\mathrm{d} 1}\right) .
$$

Eliminating $n_{j 1}$ from (14) by using (10)-(13), we obtain the dispersion relation

$$
1+\frac{G R^{2}}{\hbar^{2}} \sum_{\sigma=\mathrm{e}, \mathrm{h}} \frac{m_{\sigma}^{2} \omega_{\mathrm{p} \sigma}^{2}}{\left(k_{m}^{2}+k_{F \sigma}^{2}\right)}-\frac{G k_{m}^{2} R^{2}\left(\omega_{\mathrm{pi}}^{2}+\omega_{\mathrm{pd}}^{2}\right)}{4 \pi \omega^{2}}=0
$$

where $\omega_{\mathrm{pe}, \mathrm{ph}}=\left(4 \pi n_{\mathrm{e} 0, \mathrm{~h} 0} e^{2} / m_{\mathrm{e}, \mathrm{h}}\right)^{1 / 2}$ is the electron and hole plasma frequencies, $\omega_{\mathrm{pi}}=\left(4 \pi n_{\mathrm{i} 0} Z_{\mathrm{i}}^{2} e^{2} / m_{\mathrm{i}}\right)^{1 / 2}$ is the ion plasma frequency, $Z_{\mathrm{i}}$ is the ion charge state, and $\omega_{\mathrm{pd}}=\left(4 \pi n_{\mathrm{d} 0} Z_{\mathrm{d}}^{2} e^{2} / m_{\mathrm{d}}\right)^{1 / 2}$ is the nanoparticle plasma frequency. The wave frequency, deduced from $(15)$, is

$$
\omega=\frac{\sqrt{G} k_{m} R \omega_{\mathrm{pi}}\left(1+Z_{\mathrm{d}}^{2} m_{\mathrm{i}} n_{\mathrm{d} 0} / Z_{\mathrm{i}}^{2} m_{\mathrm{d}} n_{\mathrm{i} 0}\right)^{1 / 2}}{2 \sqrt{\pi}(1+H)^{1 / 2}},
$$

where $H=\left(G R^{2} / \hbar^{2}\right) \sum_{\sigma=\mathrm{e}, \mathrm{h}} m_{\sigma}^{2} \omega_{\mathrm{p} \sigma}^{2} /\left(k_{m}^{2}+k_{F \sigma}^{2}\right)$. 
The electrostatic wave discussed above can be driven at non-thermal level if the ions have an equilibrium axial drift $u_{0}$ against nanoparticles. Here, one should replace $\omega$ by $\omega-k u_{0}$ in (12). The resulting dispersion relation is

$$
1-\frac{G k_{m}^{2} R^{2} \omega_{\mathrm{pi}}^{2}}{4 \pi(1+H)\left(\omega-k u_{0}\right)^{2}}-\frac{G k_{m}^{2} R^{2} \omega_{\mathrm{pd}}^{2}}{4 \pi(1+H) \omega^{2}}=0 .
$$

Letting $\omega=k u_{0}+\delta$ in (17), where $\delta \ll k u_{0}$, we obtain the threshold

$$
k u_{0}=\sqrt{\frac{G}{\pi}} \frac{k_{m} R \omega_{\mathrm{pd}}}{2(1+H)^{1 / 2}},
$$

and

$$
\delta^{3}=\frac{\omega_{\mathrm{pi}}^{2}}{2 \omega_{\mathrm{pd}}^{2}} k^{3} u_{0}^{3} .
$$

Equation (19) admits an unstable solution

$$
\delta=\frac{(1+i \sqrt{3})}{2^{4 / 3}}\left(\frac{\omega_{\mathrm{pi}}}{\omega_{\mathrm{pd}}}\right)^{2 / 3} k u_{0} .
$$

The growth rate is

$$
\gamma=\frac{\sqrt{3 G} k_{m} R}{2^{7 / 3} \sqrt{\pi}(1+H)^{1 / 2}}\left(\frac{Z_{\mathrm{i}}^{2} n_{\mathrm{i} 0} m_{\mathrm{d}}}{Z_{\mathrm{d}}^{2} n_{\mathrm{d} 0} m_{\mathrm{i}}}\right)^{1 / 3} \omega_{\mathrm{pd}} .
$$

To summarize, we have investigated the dispersion property of a low-frequency electrostatic wave in a metallic dense plasma composed of degenerate electrons and holes, as well as non-degenerate ions and charged nanoparticles. Physically, the present electrostatic wave is supported by the restoring forces arising from the quantum statistical electron and hole pressures and quantum Bohm forces acting on the electrons and holes, while the inertia comes from the mass of the ions and nanoparticles. Furthermore, we have shown that the presence of an axial equilibrium ion drift (caused, say, by a dc electric field in our dense metallic plasma) against charged nanoparticles can produce a non-thermal electrostatic wave due to a two-stream instability $[27,29,30]$. From the frequency spectrum of the nonthermal electrostatic wave, one can deduce charges on nanoparticles in dense plasmas, such as those in semiconductors [31] and in thin metal films [32] with charged nanoparticles.

\section{References}

[1] Iijima, S. 1991 Nature 354, 56.

[2] Ajayan, P. M., Iijima, S. and Ichihashi, T. 1992 Phys. Rev. B. 47, 6859.

[3] Pichler, T., Knupfer, M., Golden, M. S., Fink, J., Rinzler, A. and Smalley, R. E. 1998 Phys. Rev. Lett. 80, 4729 .

[4] Sato, O., Tanaka, Y., Kobayashi, K. and Hasegawa, A. 1993 Phys. Rev. B 48, 1947.

[5] Lin, M. F. and Shung, K. W.-K. 1993 Phys. Rev. B 47, 6617.

[6] Lin, M. F., Chuu, D. S., Huang, C. S., Lin, Y. K. and Shung, K. W.-K. 1996 Phys. Rev. B 53, 15493.

[7] Jiang, X. 1996 Phys. Rev. B 54, 1387.

[8] Mowbrey, D. J., Miskovic, Z. L., Goodman, F. O. and Wang, Y. N. 2004 Phys. Rev. B 70, 195418. 
[9] Moradi, A. and Khosravi, J. 2007 Phys. Lett. A 371, 1.

[10] Wei, L. and Wang, Y. N. 2007 Phys. Rev. B 75, 193407.

[11] Guo, P. S., Chen, T., Chn, Y. W., Zhang, Z. L., Feng, T., Wang, L. L., Lin, L. F., Sun, Z. and Zhang, Z. H. 2008 Solid-State Electron. 52, 877.

[12] Sodha, M. S. and Dixit, A. 2008 J. Appl. Phys. 104, 064909.

[13] Rao, N. N., Shukla, P. K. and Yu, M. Y. 1990 Planet. Space Sci. 38, 543.

[14] Shukla, P. K. and Silin, V. P. 1992 Phys. Scripta 45, 508.

[15] Shukla, P. K., Yu, M. Y. and Bharuthram, R. 1991 J. Geophys. Res. 96, 21343.

[16] Shukla, P. K. and Rosenberg, M. 1999 Phys. Plasmas 6, 1038.

[17] Haas, F., Manfredi, G. and Feix, M. 2000 Phys. Rev. E 62, 2763.

[18] Manfredi, G. 2005 Fields Inst. Commun. 46, 263.

[19] Marklund, M., Brodin, G., Stenflo, L., Liu, C. S. 2008 Europhys. Lett. 84, 17006.

[20] Gardner, C. L. and Ringhofer, C. 1996 Phys. Rev. E 53, 157.

[21] Shukla, P. K. and Eliasson, B. 2006 Phys. Rev. Lett. 96, 245001.

[22] Shukla, P. K. and Eliasson, B. 2007 Phys. Rev. Lett. 99, 096401.

[23] Shaikh, D. and Shukla, P. K. 2007 Phys. Rev. Lett. 99, 125002.

[24] Shukla, P. K. 2006 Phys. Lett. A 352, 242.

[25] Shukla, P. K. 2009 Phys. Lett. A 373, 256.

[26] Shukla, P. K. 2009 Nature Phys. 5, 92.

[27] Shukla, P. K. and Mamun, A. A. 2002 Introduction to Dusty Plasma Physics Institute of Physics, Bristol.

[28] Jackson, J. D. 1975 Classical Electrodynamics. New York: Wiley.

[29] Shukla, P. K. and Mamun, A. A. 2003 New J. Phys. 5, 17.

[30] Shukla, P. K. and Eliasson, B. 2009 Rev. Mod. Phys. 81, 25.

[31] Markovich, P. A., Ringhofer, C. A. and Schmeiser, C. 1990 Semiconductor Equations. New York: Springer.

[32] Crouseilles, N., Hervieux, P. A. and Manfredi, G. 2008 Phys. Rev. B 78, 155412. 